\title{
Detección y asociación de Piscirickettsia salmonis en órganos y heces de salmón coho (Oncorhynchus kisutch) cultivado en el mar
}

\author{
Detection and association of Piscirickettsia salmonis in organ and faeces of coho \\ salmon (Oncorhynchus kisutch) farmed in the sea
}

\author{
Ederson Juan Montalico Pongo ${ }^{1,4}$, Héctor Rodríguez Papuico ${ }^{1}$, Julio Larenas \\ Herrera $^{2}$, Fernando Ardito Saenz ${ }^{3}$
}

\section{RESUMEN}

El objetivo de la investigación fue diagnosticar la presencia de Piscirickettsia salmonis en muestras de salmones coho (Oncorhynchus kisutch) provenientes de centros de cultivo de la Región de los Lagos, Chile, que presentaban brotes de piscirickettsiosis. Se emplearon 19 peces con signos clínicos de piscirickettsiosis. Se hicieron frotis de tejido renal, hepático, esplénico y de contenido intestinal que se procesaron mediante la prueba de inmunofluorescencia indirecta (IFI) para la detección de $P$. salmonis a través de un kit comercial. Los datos obtenidos se sometieron a la prueba de Q de Cochran y de McNemar en la distribución de Chi cuadrado. Se encontró mayor positividad en el hígado (68.4\%) y riñón (52.6\%). Además, se encontró la bacteria en las heces (47.4\%), indicando que la eliminación vía fecal del agente es posible en condiciones de cultivo. Asociando los resultados de riñón e hígado solo de logra detectar el $78.9 \%$ de los peces positivos a la bacteria, en tanto que asociando los resultados de hígado, bazo y heces se logra detectar el $100 \%$ de los peces positivos a P. salmonis.

Palabras clave: inmunofluorescencia indirecta, Piscirickettsiaceae, Salmonidae

\footnotetext{
${ }^{1}$ Universidad Nacional Jorge Basadre Grohmann, Tacna, Perú

${ }^{2}$ Universidad de Chile, Santiago, Chile

${ }^{3}$ Universidad Peruana Cayetano Heredia, Lima, Perú

${ }^{4}$ E-mail: ejmontalico@gmail.com
} 
The aim of this study was to diagnose the presence of Piscirickettsia salmonis in samples of coho salmon (Oncorhynchus kisutch) from fish farms in the Los Lagos Region, Chile, which presented outbreaks of piscirickettsiosis. In total, 19 fish with clinical signs of piscirickettsiosis were used. Smears of kidney, liver and splenic tissue and intestinal content were made, and processed using the indirect immunofluorescence test (IFAT) for the detection of $P$. salmonis through a commercial kit. The data obtained were subjected to the Cochran and McNemar Q test in the Chi square distribution. Higher positivity was found in the liver (68.4\%) and kidney (52.6\%). In addition, the bacterium was found in the faeces $(47.4 \%)$, indicating that the elimination via the faeces of the agent is possible under farming conditions. By associating the kidney and liver results, only $78.9 \%$ of the fish positive for the bacteria could be detected, while by associating the liver, spleen and faeces results, $100 \%$ of the fish positive to $P$. salmonis can be detected.

Key words: indirect immunofluorescence, piscirickettsiosis, Salmonidae

\section{INTRODUCCIÓN}

En Chile, la salmonicultura se ha convertido en uno de los sectores más exitosos de la economía nacional (Bravo y Midtlyng, 2007; SalmonChile, 2017), siendo el segundo productor mundial de salmónidos de cultivo después de Noruega (Buschmann et al., 2009; SERNAPESCA, 2016). No obstante, las enfermedades infecciosas constituyen un serio problema para esta industria, siendo el mayor impacto en la fase de engorde, efectuada en el agua de mar, generando pérdidas que exceden los 100 millones de dólares anuales (Bravo y Midtlyng, 2007; Ramírez et al., 2015). Los patógenos de mayor importancia son la bacteria Piscirickettsia salmonis y el ectoparásito Caligus spp. Según Cortés (2015), infecciones con P. salmonis son la principal causa de muerte de salmónidos en Chile, generando grandes mortalidades al final del ciclo del cultivo del salmón que dura prácticamente dos años (Rozas y Enríquez, 2014).

La piscirickettsiosis no se ha podido controlar debido a la escasa información acerca de varios aspectos del ciclo de vida de la bacteria como la virulencia y los mecanismos reguladores de patogenia (Segovia et al., 2018), así como los medios de transmisión y excreción del agente. Este patógeno también afecta diversos salmónidos como trucha arcoíris (Oncorhynchus mykiss), salmón coho (O. kisutch), salmón del Atlántico (Salmo salar), salmón chinook $(O$. tshawytscha), salmón japonés $(O$. masou) y salmón rosado (O. gorbuscha) (Leal y Woywood, 2007). Se sospecha que el salmón coho es la especie más vulnerable (OIE, 2019). P. salmonis es una bacteria intracelular facultativa, aeróbica, gram negativa, inmóvil, acapsulada, pleomórfica y difícil de cultivar (OIE, 2019; Gómez et al., 2009). Vera et al., (2012) desarrollaron un nuevo medio de cultivo líquido para $P$. salmonis. La temperatura óptima de multiplicación se encuentra entre 15 y $18{ }^{\circ} \mathrm{C}$, disminuyendo significativamente su replicación bajo los $10^{\circ} \mathrm{C}$ y sobre los $20^{\circ} \mathrm{C}$ (Fryer et al., 1992).

La bacteria infecta y sobrevive en su célula huésped mediante un proceso de virulencia regulado por una respuesta adaptativa durante la infección del huésped (Zúñiga et al., 2020). Asimismo, se describe la presen- 
cia de genes relacionados con la adquisición de hierro que codificarían posibles factores de virulencia (Pulgar et al., 2015; Yáñez et al., 2019). La piscirickettsiosis, denominada también como «Síndrome de Huito», «Síndrome del salmón coho», «Septicemia rickettsial del salmón (SRS)»»» (Leal y Woywood, 2007) es una enfermedad que se desarrolla en ambientes con agua de mar, aunque se han reportado casos en agua dulce (Bravo, 1994; Gaggero et al., 1995).

La enfermedad se presente entre abril y agosto, 6 a 12 semanas después que los «smolts» son transferidos a la fase marina del ciclo productivo (Cvitanich et al., 1990; SERNAPESCA, 2015), y se presenta en peces de todas las edades, incluyendo tamaños comerciales (Corbeil y Crane, 2009). El agente se elimina por heces, orina y bilis de individuos afectados, pudiendo penetrar piel y branquias intactas (Smith et al., 1999). Se desconoce si existen vectores marinos en la transmisión, pero la bacteria ha sido reportada en parásitos (Correal, 1995), moluscos, crustáceos, además de una diversidad de peces (Arkush et al. (2006).

Los peces infectados presentan desplazamiento errático, lento y en tirabuzón (Larenas et al., 1995, 2000; Rozas y Enriquez, 2014), letargia y anorexia (Larenas et al., 2000; Smith et al., 2011). La piel presenta úlceras hemorrágicas de diverso tamaño (Figura 1) (Branson y Nieto, 1991), con hemorragias perioculares (Larenas et al., 2000). El hematocrito se encuentra disminuido (Fryer y Hedrick, 2003). Internamente hay inflamación y necrosis en riñón, hígado, bazo, intestino, tejido hematopoyético, vejiga natatoria y musculatura esquelética (Alvarado et al., 1990; Almendras et al., 2000; Fryer y Hedrick, 2003; Monasterio, 2008).

La confirmación del patógeno en los tejidos es por aislamiento de líneas celulares (Mauel et al., 2008). P. salmonis en cultivo celular puede hacerse por la prueba de inmunofluorescencia indirecta (IFI) o la prue-

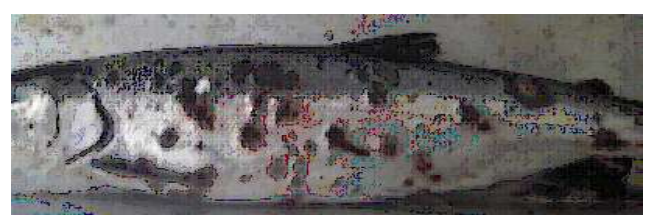

Figura 1. Pez con numerosas úlceras en piel causadas por piscirickettsiosis

ba de la reacción en cadena de polimerasa (PCR) (SERNAPESCA, 2019). La prueba de inmunofluorescencia indirecta IFI desarrollada por Lannan y Fryer (1991) es la más utilizada por ser simple, de bajo costo, rápida y de adecuada sensibilidad para detectar la bacteria. En el microscopio de fluorescencia se le observa de color verde manzana y morfología cocoide, destacándose su contorno de forma de anillo (Larenas et al., 1996). La prueba IFI incorpora como primer anticuerpo inmunoglobulina IgG de ratón anti $P$. salmonis y como segundo anticuerpo un $\mathrm{IgG}$ comercial anti-ratón conjugado con isotiocianato de fluoresceína (FITC), aunque esta técnica fue modificada por Larenas et al. (1996) mediante el uso de microondas, lo cual disminuye el tiempo para la incubación del primer y segundo anticuerpo.

El objetivo de la presente investigación fue diagnosticar la presencia, así como la asociación de Piscirickettsia salmonis en muestras de frotis renal, hígado, bazo y contenido intestinal de salmones coho provenientes de centros de cultivo, que presentaban brotes de piscirickettsiosis.

\section{Materiales y Métodos}

\section{Ubicación del Estudio y Animales}

El trabajo se realizó en el Laboratorio Nacional de Patología Animal de la Facultad de Ciencias Veterinarias y Pecuarias de la Universidad de Chile en 2015 con 19 salmo- 
Cuadro 1. Tabla de contingencia $2 \times 2$ para determinar la concordancia entre dos tratamientos en un mismo grupo de individuos

\begin{tabular}{llccc}
\hline & & \multicolumn{2}{c}{ Órgano - Heces } & \multirow{2}{*}{ Total } \\
\cline { 3 - 4 } & & Infectado & No infectado & \\
\hline \multirow{3}{*}{ Órgano - Heces } & Infectado & $\mathrm{a}$ & $\mathrm{b}$ & $\mathrm{a}+\mathrm{b}$ \\
& No infectado & $\mathrm{c}$ & $\mathrm{d}$ & $\mathrm{c}+\mathrm{d}$ \\
\cline { 2 - 5 } & Total & $\mathrm{a}+\mathrm{c}$ & $\mathrm{b}+\mathrm{d}$ & $\mathrm{a}+\mathrm{b}+\mathrm{c}+\mathrm{d}$ \\
\hline
\end{tabular}

nes coho (Oncorhynchus kisutch), adultos moribundos o muertos, con signos clínicos de piscirickettsiosis. Los especímenes provinieron de jaulas de crianza de salmones de la Región de los Lagos, Chile, zona endémica, cuyo diagnóstico fue realizado por la empresa salmonera, según protocolos y normativa del SERNAPESCA (2018).

\section{Necropsia y Frotis}

Se procedió a la eutanasia de los peces con un golpe en la cabeza. La necropsia se realizó según Acuña et al. (2014). Se hizo un corte abdominal ventral con bisturí, del opérculo hasta el ano, un segundo corte de la zona anal hasta el extremo superior del opérculo y un tercer corte uniendo los dos cortes previos a finde facilitar la toma de muestras y evitar la contaminación cruzada. Se hicieron frotis de tejido renal, hígado, bazo y contenido fecal. En el caso de órganos, se hizo un corte y se introdujo un hisopo en la zona de la incisión, mientras que para las heces, se hizo el corte longitudinal en el intestino. El transporte de las muestras desde el centro de cultivo al laboratorio se hizo en un contenedor con bolsas de hielo.

\section{Análisis de las Muestras}

Para el análisis de las muestras se empleó la técnica IFI establecido por Lannan et al. (1991). Se utilizó el kit comercial SRS-
Fluoro Test Indirecto del laboratorio BiosChile para detectar $P$. salmonis. El procesamiento de las muestras se realizó utilizando el protocolo del kit.

Como controles positivos se usaron frotis de una alícuota de cultivo celular CHSE214 infectada con P. salmonis (100\% de efecto citopático). Para confirmar la presencia del agente se realizaron pruebas de tinción Giemsa, Gram, además de las pruebas de diagnóstico IFI y PCR. Los controles negativos utilizados fueron frotis de tejidos de riñón, hígado, bazo y contenido intestinal de truchas arcoíris, clínicamente sanas, de la Región de Valparaíso, Chile, lugar donde nunca hubo se ha registrado brotes de piscirickettsiosis. Se comprobó el estatus de estos controles mediante pruebas de Gram, IFI y PCR. Asimismo, como control negativo se utilizó frotis de cultivos celulares libres de la bacteria. Tanto los controles positivos como los negativos procedieron del laboratorio de Patología de la Facultad de Medicina Veterinaria de la Universidad de Chile.

Para observar al patógeno se usó el microscopio de epifluorescencia Nikon, Optiphot-2, Tokio Japón, con longitud de onda de $494 \mathrm{~nm}$. En cada muestra se observaron 10 campos con un aumento de $1000 \mathrm{X}$, según lo descrito por Smith et al. (1999). Las muestras se clasificaron como negativas (-) o po- 
Cuadro 2. Detección de Piscirickettsia salmonis en frotis de riñón, hígado, bazo y heces de 19 individuos salmón coho (Oncorhynchus kisutch), procedentes de la Región de los Lagos, Chile

\begin{tabular}{|c|c|c|c|c|c|}
\hline \multirow{2}{*}{ Pez } & \multicolumn{4}{|c|}{ Órganos } & \multirow{2}{*}{$\begin{array}{c}\text { Órganos } \\
\text { positivos }(n)\end{array}$} \\
\hline & Riñón & Hígado & Bazo & Heces & \\
\hline 1 & - & - & + & - & 1 \\
\hline 2 & + & - & + & + & 3 \\
\hline 3 & + & + & - & - & 2 \\
\hline 4 & - & + & - & - & 1 \\
\hline 5 & + & + & - & + & 3 \\
\hline 6 & - & - & - & + & 1 \\
\hline 7 & - & - & - & + & 1 \\
\hline 8 & + & + & - & - & 2 \\
\hline 9 & + & + & - & - & 2 \\
\hline 10 & + & + & - & - & 2 \\
\hline 11 & - & + & - & + & 2 \\
\hline 12 & - & + & - & - & 1 \\
\hline 13 & - & + & + & - & 2 \\
\hline 14 & - & - & - & + & 1 \\
\hline 15 & + & + & - & - & 2 \\
\hline 16 & - & + & + & + & 3 \\
\hline 17 & + & + & - & + & 3 \\
\hline 18 & + & + & - & - & 2 \\
\hline 19 & + & - & - & + & 2 \\
\hline Total positivos & 10 & 13 & 4 & 9 & \\
\hline Total negativos & 9 & 6 & 15 & 10 & \\
\hline Positivos (\%) & 52.6 & 68.4 & 21.1 & 47.4 & \\
\hline
\end{tabular}

sitivas (+) si se detectó al menos una bacteria dentro de los 10 campos visuales.

\section{Análisis Estadístico}

Se utilizó la prueba Q de Cochran y y de McNemar en la distribución de Chi cuadrado para comparar la presencia de $P$. salmonis en órganos y heces. Los resultados se ordenaron en filas y columnas, donde los resultados (+) se asignó el 1 y a los (-) el
0 . La hipótesis nula (Ho) indicó la probabilidad de respuesta $(+)$ para cada una de las cuatro muestras y la hipótesis alterna $(\mathrm{Ha})$ indicó la probabilidad de registrar una respuesta $(+)$ para cada una de las cuatro muestras es diferente. Se utilizó la fórmula de Siegel (1956): $\times^{2}=\left((\mathrm{K}-1)\left[\mathrm{K} \ll \mathrm{Gn}^{2}-(\ll \mathrm{Gn})^{2}\right]\right) / \mathrm{K}$ $\ll \mathrm{Lc}-\ll \mathrm{Lc}^{2}$, donde $\mathrm{X}_{\mathrm{Q}}^{2}$ : Estadígrafo Chi cuadrado de la prueba $\mathrm{Q}$ de Cochran; $\mathrm{K}: \mathrm{N}^{\circ}$ de tratamientos; Gn: $\mathrm{N}^{\circ}$ de respuestas de cambio $(+)$ de cada tratamiento en la columna; 


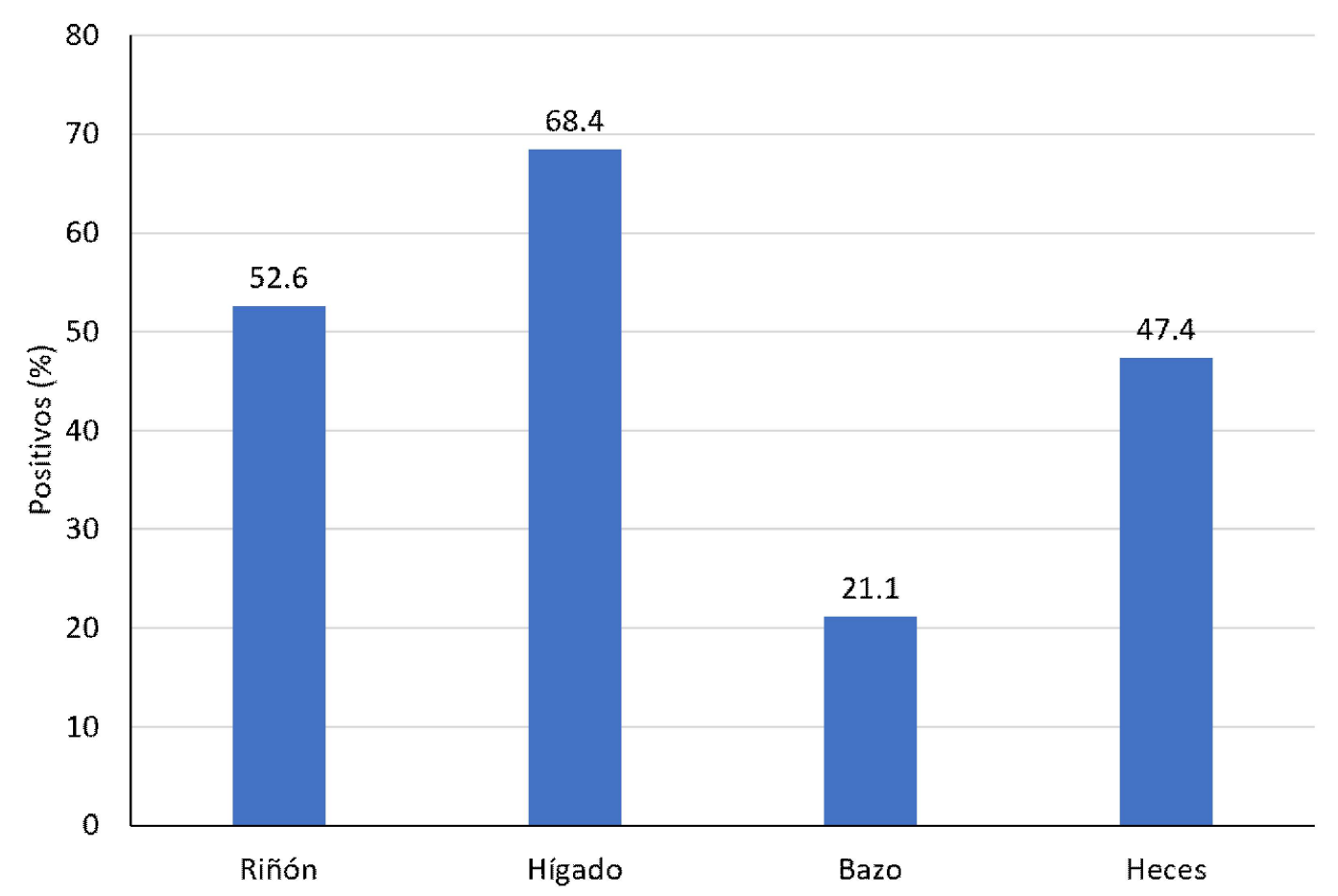

Figura 2. Porcentaje de positividad de Piscirickettsia salmonis en frotis de órganos y heces observados al microscopio de epifluorescencia de 19 especímenes de salmón coho (Oncorhynchus kisutch), procedentes de la Región de los Lagos, Chile

Lc: $\mathrm{N}^{\circ}$ de respuestas de cambio $(+)$ por individuo de la muestra.

Para determinar la asociación estadística de $P$. salmonis en órganos y heces, se utilizó el método McNemar $\left(\mathrm{X}^{2}\right)$ (DawsonSaunders y Trapp, 1997; Martin et al., 1997), permitiendo evaluar con á $=0.05$ la concordancia entre dos tratamientos en un mismo grupo de individuos. Se usó una tabla de contingencia agrupados en pares, según lo señalado por Martin et al. (1997) (Cuadro 1). La fórmula empleada fue $\times{ }_{1}^{2}=(|\mathrm{b}-\mathrm{c}|-1)^{2} / \mathrm{b}+\mathrm{c}$, donde a: Los órganos detectados como $(+)$ por la prueba IFI y que son verdaderamente positivos; b: Los órganos de individuos falsos $(+)$. IFI los define como $(+)$, pero en realidad son (-); c: Los órganos de individuos falsos (). IFI los define como (-), pero en realidad son (+); d: Órganos de individuos detectados como (-) por IFI y que son verdaderamente $(-) ; \mathbf{a}+\mathbf{c}$ : Total de órganos verdaderamente positivos; $\mathbf{b}+\mathbf{d}$ : Total de órganos verdaderamente negativos.

\section{Resultados}

Se confirmó la presencia de lesiones macroscópicas correspondientes a piscirickettsiosis en todos los especímenes. Se observaron úlceras prominentes en la piel, palidez hepática y hemorragias en órganos abdominales. Los resultados de la prueba de IFI se muestran en el Cuadro 2 y la Figura 2). En el cuadro 2 se puede observar que todos los peces resultaron positivo a la presencia de $P$. salmonis, pues se logró detectar la presencia del agente en al menos una muestra de órganos o en heces. 
Cuadro 3. Resumen de la asociación encontrada entre órganos y heces de 19 especímenes de salmón coho (Oncorhynchus kisutch), procedentes de la Región de los Lagos, Chile salmón coho, afectados positivos a Piscirickettsia salmonis

\begin{tabular}{cccc}
\hline & Hígado & Bazo & Heces \\
\hline Riñón & Existe asociación & Existe asociación & Existe asociación \\
& significativa & significativa & significativa \\
& $\left(\mathrm{X}^{2}=0.333\right)$ & $\left(\mathrm{X}^{2}=2.083\right)$ & $\left(\mathrm{X}^{2}=0.307\right)$ \\
Hígado & & No existe asociación & Existe asociación \\
& & significativa & significativa \\
& & $\left(\mathrm{X}^{2}=4.923\right)$ & $\left(\mathrm{X}^{2}=0.642\right)$ \\
Bazo & & Existe asociación \\
& & & significativa \\
& & & $\left(\mathrm{X}^{2}=1.777\right)$ \\
\hline
\end{tabular}

Grado de asociación entre órganos y heces en forma conjunta

Empleando la tabla de contingencia del Cuadro 1 y la prueba Q de Cochram con un á $=5 \%$ con la distribución de Chi cuadrado y grados de libertad $\mathrm{K}-1=3$ se obtiene la sumatoria de calores positivos por cada órgano y heces; es decir, 10, 13, 4 y 9 respectivamente, lo que resulta en un valor de $\mathrm{Q}=$ 7.636, el cual indica que los datos obtenidos difieren significativamente al ser analizados en forma conjunta.

Asociación entre órganos y heces

El Cuadro 3 muestras el resultado de las asociaciones estadísticas entre órganos y heces de salmón coho usando la ecuación de $\operatorname{McNemar}\left(\mathrm{X}^{2}\right)$.

\section{Discusión}

Se analizaron muestras de 19 salmones coho con la prueba IFI para detectar $P$. salmonis, comprobando mayor positividad al patógeno en las muestras de hígado (68.4\%), seguido de riñón $(52.6 \%)$, heces $(47.4 \%)$ y bazo (21.1\%) (Figura 2), demostrando que ningún órgano en forma independiente es capaz de detectar el $100 \%$ de los animales con piscirickettsiosis. $\mathrm{Al}$ asociar los resultados de los órganos en pares y de a tres se pudo establecer la mejor asociación capaz de detectar el $100 \%$ de los animales infectados (Figura 3).

Según la Figura 3, la asociación de riñón solo detecta el $78.9 \%$ de peces positivos a $P$. salmonis, mientras que la asociación de hígado, bazo y heces detectó el 100\% de infectados. Esto no concuerda con el OIE, que indica que los mejores órganos para diagnosticar la piscirickettsiosis son el riñón e hígado (Evensen, 2016).

Según la OIE (2006) se menciona que los órganos de diagnóstico para piscirickettsiosis son solo el riñón y el hígado. No obstante, en el presente trabajo se incluyeron muestras de bazo, debido a que Cvitanich et al. (1990) describe que rickettsias como $P$. salmonis afectan princi- 


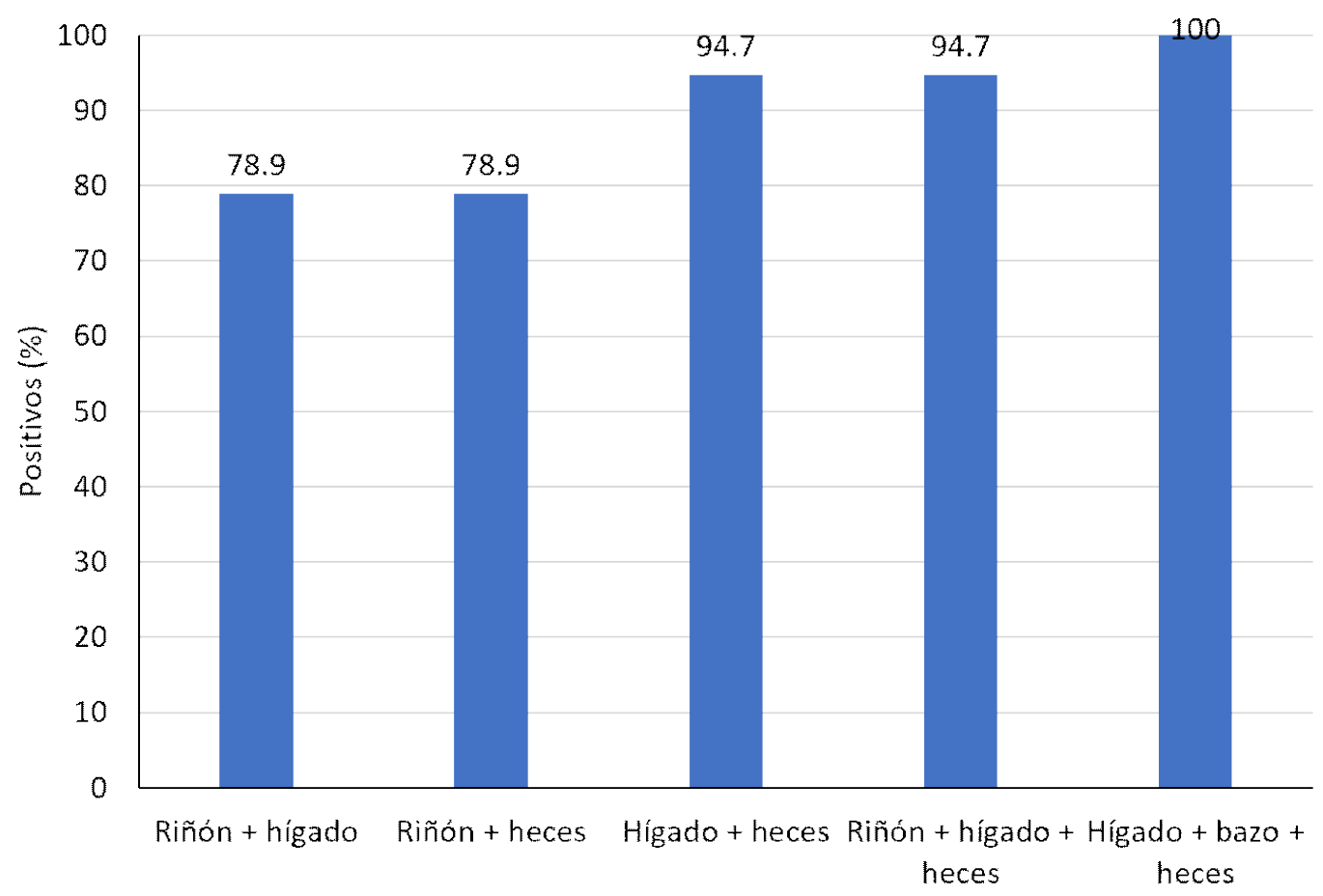

Figura 3. Porcentaje de detección de piscirickettsiosis (Piscirickettsia salmonis) por asociación de +órganos-heces de 19 especímenes de salmón coho (Oncorhynchus kisutch), procedentes de la Región de los Lagos, Chile

palmente al bazo y riñón. De otra parte, se decidió incluir muestras de heces porque Smith et al. (1999) describieron que el agente es eliminado por vía fecal.

En el presente estudio se encontró que la lesión macroscópica más prominente eran las úlceras, aunque hasta la fecha no se ha demostrado que las úlceras sea otra vía de eliminación del agente. Salinas et al. (1998) demostró que las vías de eliminación de $P$. salmonis en peces con signología de piscirickettsiosis era la orina y la bilis. Asimismo, se ha demostrado la eliminación de $P$. salmonis por vía fecal al medio marino (Salinas et al., 1998; Larenas et al., 2005; Peirano, 2015), pero se debe establecer si el patógeno es viable y logra afectar a otras especies susceptibles. En el presente estudio se demostró que los peces excretan P. salmonis vía fecal en condiciones de cultivo en agua de mar.
Según Allen y Hopeb (2014), hay bacterias que afectan a salmónidos y no salmónidos, capaces de sobrevivir en el estómago para luego crecer y multiplicarse en el intestino, como el Vibrio anguillarum, agente causal de la vibriosis en salmónidos. Este soporta el pH y las enzimas del estómago, luego se desarrolla en el intestino y se elimina vía fecal; no obstante, no hay estudios que indiquen que $P$. salmonis sea capaz de infectar por esta vía a otros peces. Salinas et al. (1998) y Larenas et al. (2005) demostraron la presencia de la bacteria en riñón, heces, orina y bilis en truchas arcoíris, previamente infectadas y cultivadas en agua dulce. Según esto, la bacteria podría ingresar al intestino y ser eliminada vía fecal por medio de la bilis. En el presente estudio se halló positividad al agente en el riñón, estimándose que la bacteria podría ser eliminada en la orina, vía riñón, tal y como fue reportado por Henríquez et 
al. (2016), por lo que sería oportuno estudiar el riñón y orina de salmónidos cultivados en agua de mar y hallar la asociación del agente y la posible eliminación vía renal y establecer si los salmones clínicamente sanos logran eliminar P. salmonis vía fecal.

Los resultados sobre presencia del $P$. salmonis en hígado, bazo y heces aquí hallados, muestran importante avance y ayuda, dando mayor precisión diagnóstica de la infección. Los resultados confirman la eliminación de $P$. salmonis por las heces en salmones coho cultivado en agua de mar, lo cual solo se había observado de forma experimental (Larenas et al., 2005). Se podría considerar que la bacteria es viable y, por lo tanto, existiría el riesgo de transmisión horizontal hacia otras especies susceptibles. Además, la excreción del agente vía fecal puede representar un medio de detección temprana, antes que aparezca un brote de piscirickettsiosis que afecte a los centros de cultivo en Chile.

\section{Conclusiones}

- $\quad$ Se detectó Piscirickettsia salmonis mediante la prueba de inmunofluorescencia indirecta (IFI) en hígado, riñón bazo y heces del salmón coho (Oncorhynchus kisutch), cultivados en agua de mar.

- Se encontró mayor frecuencia del agente en el hígado (68.4\%) seguido del riñón $(52.6 \%)$, indicando que ninguno de los dos órganos fue suficiente para detectar el total de peces positivos a $P$. salmonis.

- La asociación de los resultados de frotis de muestras de hígado, bazo y heces permite detectar el $100 \%$ de los peces infectados con la bacteria.

- $\quad$ P. salmonis se encontró por primera vez en heces de salmón coho provenientes de un cultivo en agua de mar.

\section{Literatura Citada}

1. Acuña M, Larenas $J$, Olivares $R$. 2014. Manual de anatomía y procedimientos diagnósticos en salmónidos. Santiago, Chile: Servicio Nacional de Pesca y Acuicultura. $17 \mathrm{p}$.

2. Allen SA, Hopeb TJ. 2014. Mechanisms of immunoglobulin-mediated mucus entrapment of pathogens at various mucosal surfaces. Antibody Fc 1: 351-357. doi: 10.1016/B978-0-12394802-1.00020-0

3. Almendras FE, Fuentealba IC, Frederick Markham RF, Speare DJ. 2000. Pathogenesis of liver lesions caused by experimental infection with Piscirickettsia salmonis in juvenile Atlantic salmon Salmo salar L. J Vet Diagn Invest 12: 552-557. doi: 10.1177/ 104063870001200610

4. Alvarado V, Schafer W, Enriquez, $R$, Monrás M, Cubillos V, Farías C, Alberdi A. 1990. Síndrome del salmón coho (S.S.C.), nueva enfermedad de salmonídeos cultivados en fase de agua de mar en Chile. Situación actual. Pat Anim 4: 10-13.

5. Arkush KD, Edes HL, McBride AM, Adkison MA, Hedrick RP. 2006. Persistence of Piscirickettsia salmonis and detection of serum antibodies to the bacterium in white seabass Atractoscion nobilis following experimental exposure. Dis Aquat Org 73: 131-139. doi: 10.3354/ dao073131

6. Branson EJ, Diaz-Muñoz, DN. 1991. Description of a new disease condition occurring in farmed coho salmon, Oncorhynchus kisutch, in South America. J Fish Dis 14:147-156. doi: 10.1111/j.1365-2761.1991.tb00585.x

7. Bravo S. 1994. Piscirickettsiosis in freshwater. Bull Eur Assn Fish P 14:137-138.

8. Bravo S, Midtlyng PJ. 2007. The use of fish vaccines in the Chilean salmon industry 1999- 2003. Aquaculture 270: 36-42. doi: 10.1016/j.aquaculture.2007.06.017 
9. Buschmann AH, Cabello F, Young K, Carvajal J, Varela DA, Henriquez $L$. 2009. Salmon aquaculture and coastal ecosystem health in Chile: analysis of regulations, environmental impacts and bioremediation systems. Ocean Coast Manage 52: 243-249. doi: 10.1016/ j.ocecoaman.2009.03.002

10. Corbeil S, Crane M. 2009. Piscirickettsia salmonis. [Internet]. Available in: https://www.agriculture.gov.au/sites/ default/files/sitecollectiondocuments/animal/ahl/ANZSDP-Piscirickettsiasalmonis.pdf

11. Correal P. 1995. Prospección de Piscirickettsia salmonis en fauna marina silvestre asociada al cultivo intensivo en salmónidos. Tesis de Médico Veterinario. Santiago, Chile: Univ. de Chile. 62 p.

12. Cortés M. 2015. Study of the type $4 \mathrm{~b}$ protein secretion system $(\mathrm{dot} / \mathrm{icm})$ and the effector protein SdhA in Piscirickettsia salmonis. Tesis Doctoral. Puerto Montt, Chile: Univ. Austral de Chile. $72 \mathrm{p}$.

13. Cvitanich J, Gárate O, Smith C. 1990. Etiological agent in a Chilean coho disease isolated and confirmed by Koch's postulates. AFS/FHS Newsletter 18: 1-2.

14. Dawson-Saunders B, Trapp R. 1997. Bioestadística médica. Mexico: El Manual Moderno. $435 \mathrm{p}$.

15. Fryer JL, Hedrick RP. 2003. Piscirickettsia salmonis: a Gram-negative intracellular bacterial pathogen of fish. J Fish Dis 26: 251-262. doi: 10.1046/j.13652761.2003.00460.x

16. Fryer J, Lannan C, Giovannoni S, Wood N. 1992. Piscirickettsia salmonis gen. nov., sp. nov., the causative agent of an epizootic disease in salmonid fishes. Int J Syst Bacteriol 42: 120-126. doi: 10.1099/00207713-42-1-120
17. Gaggero A, Castro H, Sandino A. 1995. First isolation of Piscirickettsia salmonis from coho salmon, Oncorhynchus kisutch (Walbaum), and rainbow trout, Oncorhynchus mykiss (Walbaum), during the freshwater stage of their life cycle. J Fish Dis 18: 277279. doi: $10.1111 /$ j. 1365-2761.1995.tb00303.x

18. Gómez F, Henríquez V, Marshall SH. 2009. Additional evidence of the facultative intracellular nature of the fish bacterial pathogen Piscirickettsia salmonis. Arch Med Vet 41: 261-267. doi: 10.4067/S0301-732X2009000300011.

19. Henríquez P, Kaiser M, Bohle H, Bustos P, Mancilla M. 2016. Comprehensive antibiotic susceptibility profiling of Chilean Piscirickettsia salmonis field isolates. J Fish Dis 39: 441-448. doi: 10.1111/jfd. 12427

20. Lannan CN, Fryer J. 1991. Recommended methods for inspection of fish for the salmonis rickettsia. Bull Eur Assn Fish P 11: 135-136.

21. Larenas J, Contreras J, Smith P. 2000. Piscirickettsiosis: uno de los principales problemas en cultivos de salmones en Chile. Tecnovet 6: 28-30.

22. Larenas J, Hidalgo L, Garcés $H$, Fryer J, Smith P. 1995. Piscirickettsiosis: lesiones en salmón del Atlántico (Salmo salar) infectados naturalmente con Piscirickettsia salmonis. Av Cs Vet 10: 53-58. doi: 10.5354/0716-260X.1995.10440

23. Larenas J, Astorga C, Contreras J, Garcés H, Fryer JL, Smith P. 1996. Rapid detection of Piscirickettsia salmonis using microwave irradiation. Fish Pathol 31: 231-232. doi: https:// doi.org/10.3147/jsfp.31.231

24. Larenas J, Zamorano E, Smith P. 2005. Detección de Piscirickettsia salmonis en heces de alevines de salmón coho (Oncorhynchus kisutch) infectados por transmisión vertical. Mon Electr Patol Vet 2: 59-67. 
25. Leal J, Woywood D. 2007. Piscirickettsia en Chile: avances y perspectivas para su control. Salmociencia 2: 34-42.

26. Martin ER, Kaplan NL, Weir BS. 1997. Tests for linkage and association in nuclear families. Am J Hum Genet 61: 439-448. doi: 10.1086/514860

27. Mauel M, Ware C, Smith P. 2008. Culture of Piscirickettsia salmonis on enriched blood agar. J Vet Diagn Invest 20:213-214. doi: 10.1177/104063870802000211

28. Monasterio M. 2008. Descripción patológica en salmones coho (Oncorhynchus kisutch) coinfectados para reproducir experimentalmente piscirickettsiosis y anemia hemolítica del salmón. Tesis de Médico Veterinario. Santiago, Chile: Univ. de Chile. 98 p.

29. [OIE] Organización Mundial de Sanidad Animal. 2019. Manual de pruebas de diagnóstico para los animales acuáticos. Paris: OIE. [Internet]. Disponible en: https://www.oie.int/es/normas/ manual-acuatico/acceso-en-linea/

30. Peirano P. 2015. Determinación de la presencia y asociación de Piscirickettsia salmonis en heces, hígado y riñón de trucha arcoíris (Oncorhynchus mykiss) en condiciones de cultivo en mar. Tesis de Médico Veterinario. Santiago, Chile: Univ. de Chile. 28 p.

31. Pulgar R, Travisany D, Zuñiga A, Maass A, Cambiazo V. 2015. Complete genome sequence of Piscirickettsia salmonis LF-89 (ATCC VR-1361) a major pathogen of farmed salmonid fish. J Biotechnol 212: 30-31. doi: 10.1016/ j.jbiotec.2015.07.017

32. Ramirez R, Gomez F, Marshall SH. 2015. The infection process of Piscirickettsia salmonis in ûsh macrophages is dependent upon interaction with host-cell clathrin and actin. FEMS Microbiol Lett 362: 1-8. doi: 10.1093/femsle/fnu012

33. Rozas M, Enríquez R. 2013. Piscirickettsiosis and Piscirickettsia salmonis in fish: a review. J Fish Dis 37: 163-188. doi: $10.1111 /$ jfd.12211
34. Salinas G, Contreras J, Smith P, Larenas $J$. 1998. Horizontal transmission and excretion of Piscirickettsia salmonis in rainbow trout (Oncorhynchus mykiss) in fresh water condition. In: VIII International Conference «Diseases of Fish and Shellûsh». Edinburgh, UK.

35. SalmonChile. 2017. Producción. Santiago de Chile: SalmonChile. [Internet]. Disponible en: https://www.salmonchile.cl/produccion-salmonchile/

36. Segovia C, Arias-Carrasco R, Yañez AJ, Maracaja-Coutinho V, Santander J. 2018. Core non-coding RNAs of Piscirickettsia salmonis. Plos One 13: e0197206. doi: 10.1371/ journal.pone.0197206

37. [SERNAPESCA] Servicio Nacional de Pesca y acuicultura. 2015. Anuario estadístico de pesca 2014: Subsector Acuicultura. Valparaíso, Chile: Servicio Nacional de Pesca y acuicultura.

38. [SERNAPESCA] Servicio Nacional de Pesca y acuicultura. 2016. Informe sanitario de salmonicultura en Centro Marinos año 2015. Santiago, Chile: Servicio Nacional de Pesca y acuicultura. [Internet]. Disponible en: http:// www.sernapesca.cl/sites/default/files/ informe_sanitario_2015.pdf

39. [SERNAPESCA] Servicio Nacional de Pesca y acuicultura. 2019. Informe sanitario de salmonicultura en Centro Marinos año 2018. Santiago, Chile: Servicio Nacional de Pesca y acuicultura. [Internet]. Disponible en: http:// www.sernapesca.cl/sites/default/files/ informe_sanitario_salmonicultura_en_centros_marinos_2018_final.pdf

40. [SERNAPESCA] Servicio Nacional de Pesca y Acuicultura. 2018. Manual de inocuidad y certificación. Valparaíso, Chile: Servicio Nacional de Pesca y acuicultura. [Internet]. Disponible en: http://www.sernapesca.cl/sites/default/ files/parte_i_introduccion_version_10.07.18.pdf 
41. Siegel S. 1956. Nonparametric statistics for the behavioral sciences. New York, USA: McGraw Hill. 312 p.

42. Smith P, Pizarro P, Ojeda P, Contreras J, Oyanedel S, Larenas J. 1999. Routes of entry of Piscirickettsia salmonis in rainbow trout (Oncorhynchus mykiss). Dis Aquat Org 37: 165172. doi: 10.3354/dao037165

43. Vera T, Isla A, Cuevas A, Figueroa J. 2012. Un nuevo medio de cultivo líquido para el patógeno Piscirickettsia salmonis. Arch Med Vet 44: 273-277. doi: 10.4067/S0301-732X2012000300010

44. Yáñez JM, Yoshida GM, Parra A, Correa $K$, Barria A, Bassini $L N$, Christensen K, et al. 2019. Compara- tive genomic analysis of three salmonid species identifies functional candidate genes involved in resistance to the intracellular bacterium Piscirickettsia salmonis. Front Genet 10: 665. doi: 10.3389/fgene. 2019.00665

45. Zúñiga A, Aravena P, Pulgar R, Travisany D, Ortiz-Severín J, Chávez. F, Maas A. 2020. Transcriptomic changes of Piscirickettsia salmonis during intracellular growth in a salmon macrophage-like cell line. Front Cell Infect $\mathrm{Mi}$ 9: 426. doi: 10.3389/ fcimb. 2019.00426 